\title{
Application of Finite Element Power Flow and Visualization in Rolling Mill Vibration
}

\author{
Biao Xiao ${ }^{1}$, Xiaoqiang Yan $^{1, *}$ \\ ${ }^{1}$ School of Mechanical Engineering, University of Science and Technology Beijing, 30 Xueyuan Road Haidian District Beijing, China
}

\begin{abstract}
The power flow harmonic responses of the upper roll system are obtained by combining the speed solution and stress solution, and compare with the traditional harmonic response, we find that the traditional vibration displacement, velocity and acceleration harmonic response spectrum can't completely reflect the reality of the vibration energy. The power flow vectors of the mill can be obtained with a color vector program developed independently. Compared with the vibration displacement vectors, we found that the displacement vector distribution of the mill is quite different from that of the power flow vector with the frequency of $104 \mathrm{~Hz}$ : the displacement vector amplitude gradually increases from the bottom to the top of the mill, and the displacement vector is transmitted from bottom to top vertically. The power flow vector amplitude of the working roll and the intermediate roll is larger than that of the other parts, and power flow vectors present complex transfer directions. Similarly, when the considering frequency is taken as other values, the distribution of vibration displacement vector and power flow vector are also variable.
\end{abstract}

\section{Introduction}

Power flow has been defined for half a century, and then continuously improved. Initially, Lyon and Madanik used power flow to analyze the vibration transmission of a simple system [1]. Goyder and White introduced the concept of power flow, and applied it on flexible onedimensional and two-dimensional structure [2-4]. Pinnington and White also used this method to analyze infinite structures in detail [5].

After the perfection of power flow theory, many scholars in China applied power flow to their respective fields, included: Kongjie Song [6] commented on the application of power flow theory in flexible vibration control technology, and prospected its development trend. Bin Xiao and Biao Li [7] divided the double-layer vibration isolation system into sub-structure systems, established the transfer matrix, and then used the average vibration energy as the objective function to synthesize the transfer relationship of the system by power flow method to study the vibration transmission characteristics of the passive vibration isolation process. Weibo Zhang, Junchuan Niu et al [8] established the mobility matrix formulas for multi-substructure coupling system with multi-source excitation, which could fully consider the flexibility of vibration source subsystem and deal with the coupling between multi-source disturbances. Xiaole Wang, Lingling Sun et al [9] established the power flow transfer equation of the whole cylindrical shell foundation vibration isolation system by using the substructure mobility method. Yaoquan Cai, Fanming Zeng et al [10] used finite element power flow to analyze the influence of transmission characteristics of supporting bearings on coupling vibration of shafting-base-shell system under various working conditions. Na Fu, Yu Liu et al [11] used power flow to study the vibration energy characteristics of double-block ballastless damping track, discussed the vibration energy redistribution of the track, introduced the corresponding power flow criteria, and evaluated the vibration of track structure. Jinlin Liu, Guojun Lai et al. [12] used the finite element power flow to analyze the influence of the thrust bearings on the vibration of the shafting-base-hull coupling system, and the relationship between the power flow through the transmission path, and the underwater radiated noise of the system was discussed.

This paper introduces the finite element power flow and visualizes it to research the rolling mill vibration. The difference between vibration velocity harmonic response and the power flow harmonic response is presented in chapter 3 , and the power flow vector of the rolling mill is compared with the displacement vector of the rolling mill in chapter 4 .

\section{Mathematical model}

\subsection{Finite element power flow}

The definition of power flow of solid element is given in reference [13], A 8-node hexahedral cube element is shown in Fig.1. 


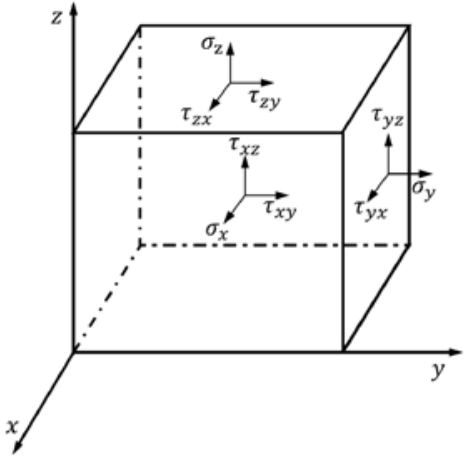

Fig. 1. Hexahedral element

Assuming that the global coordinate system of the unit in Fig. 1 is XYZ, the power flow of the unit in three directions under the global coordinate is:

$$
\left\{\begin{array}{l}
P_{x}=-\frac{\omega}{2} \operatorname{Im}\left(\sigma_{x} u_{x}^{*}+\tau_{x y} u_{y}^{*}+\tau_{x z} u_{z}^{*}\right) \\
P_{y}=-\frac{\omega}{2} \operatorname{Im}\left(\tau_{x y} u_{x}^{*}+\sigma_{y} u_{y}^{*}+\tau_{y z} u_{z}^{*}\right) \\
P_{z}=-\frac{\omega}{2} \operatorname{Im}\left(\tau_{x z} u_{x}^{*}+\tau_{y z} u_{y}^{*}+\sigma_{z} u_{z}^{*}\right)
\end{array}\right.
$$

where $P_{x}, P_{y}$ and $P_{z}$ are the directional power flow of the unit in Fig.1, respectively; $\sigma_{x}, \sigma_{y}$ and $\sigma_{z}$ are the directional stress, respectively; $\tau_{x y}, \tau_{y z}$ and $\tau_{x z}$ are the shear stress of plane-xy, plane-xz and plane-yz, respectively; $u_{x}^{*} 、 u_{y}^{*}$ and $u_{z}^{*}$ are the directional conjugate form of displacement, $\omega$ is the circular frequency.

Then we have the total power flow of the unit:

$$
P_{0}=\sqrt{p_{x}^{2}+p_{y}^{2}+p_{z}^{2}}
$$

In order to observe the flow direction of power flow, the power flow is defined as vectors and expressed as follow:

$$
\begin{aligned}
\boldsymbol{P}_{x} & =\left[\begin{array}{lll}
P_{x} & 0 & 0
\end{array}\right] \\
\boldsymbol{P}_{y} & =\left[\begin{array}{lll}
0 & P_{y} & 0
\end{array}\right] \\
\boldsymbol{P}_{z} & =\left[\begin{array}{lll}
0 & 0 & P_{z}
\end{array}\right] \\
\boldsymbol{P} & =\boldsymbol{P}_{x}+\boldsymbol{P}_{y}+\boldsymbol{P}_{z}
\end{aligned}
$$

In finite element power flow analysis, the whole power flow can be regarded as the combination of the power flow of each element. The power flow and its direction can be directly obtained by solving the displacement and stress on each node of the model.

\subsection{Simulation model and parameter setting}

The F5 stand of 1550 cold tandem mill is taken as the simulation object, and the whole mill is assumed to be composed of homogeneous and pure elastic materials. The solid model and finite element mesh model are shown in Fig.2.

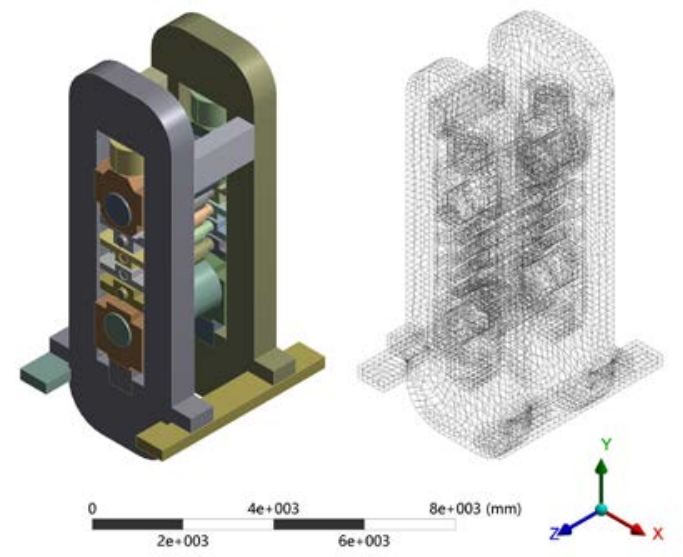

Figure 2. Mill model and grid diagram

The relevant parameters of the model in Fig.2 are shown in Tab.1.

Table1. Mill model parameters

\begin{tabular}{|c|c|}
\hline Parameter & Value \\
\hline Elastic Modulus (Pa) & $2.01 \times 10^{11}$ \\
\hline Poisson's ratio & 0.3 \\
\hline Number of elements & 25720 \\
\hline Number of nodes & 89079 \\
\hline Density $\left(\mathrm{kg} / \mathrm{m}^{3}\right)$ & 7800 \\
\hline
\end{tabular}

Most of the parts of the rolling mill are made of structural steel, thus, we assume that the material of the mill model is structural steel. The rolling mill model is divided by hexahedral grid. In addition, the mill model is simplified, and the chamfering of each part is removed on the premise of not affecting the quality of the model and the overall shape.

\section{Power flow harmonic response}

\subsection{Harmonic response of power flow and vibration speed}

The pad at the bottom of the rolling mill is set as the full constraint, and the uniform load excitation is set on the roll gap. The excitation force is $F=F_{0}+F_{1} \sin (\omega t+\theta)$, where $F_{0}$ is the average rolling force amplitude, $F_{0}=$ $2 \times 10^{7} \mathrm{~N}, F_{1}$ is the fluctuating rolling force, $F_{1}=2 \times$ $10^{6} \mathrm{~N}, \theta$ is the phase angle, $\theta=0$, and the excitation direction is the $\mathrm{y}$-axis direction. The harmonic response analysis is carried out by using ANSYS. The vibration velocity harmonic response spectra and the power flow harmonic response spectra of the upper working roll, upper intermediate roll and upper support roll in Y-axis direction are obtained respectively. For the convenience of analysis and comparison, put these two spectra in the same diagram, as shown in Fig. $3 \sim$ Fig. 5 . 


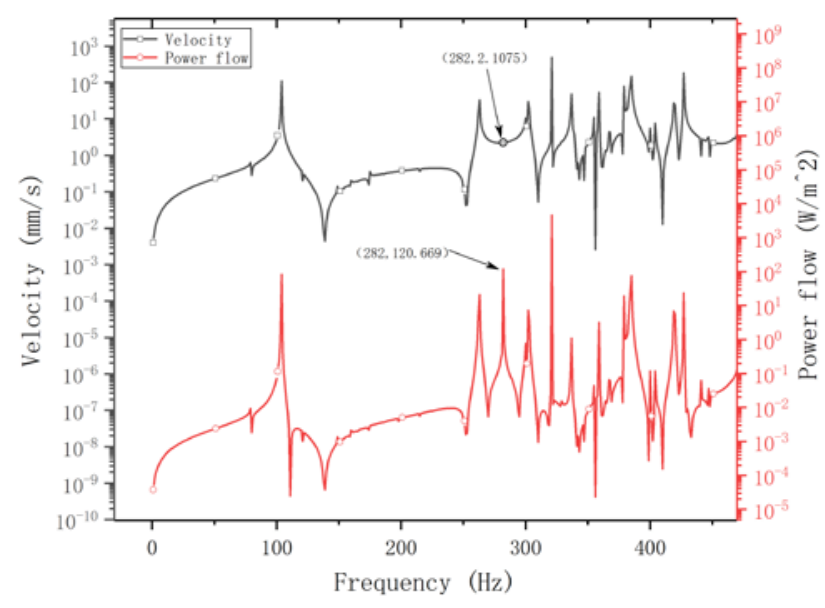

Fig. 3. Vibration velocity and power flow of the working roll

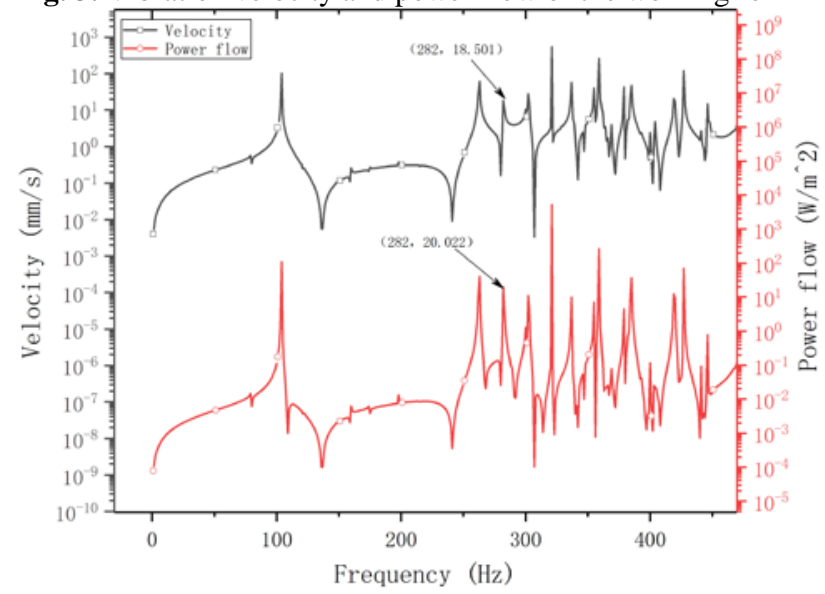

Fig. 4. Vibration velocity and power flow of the intermediate roll

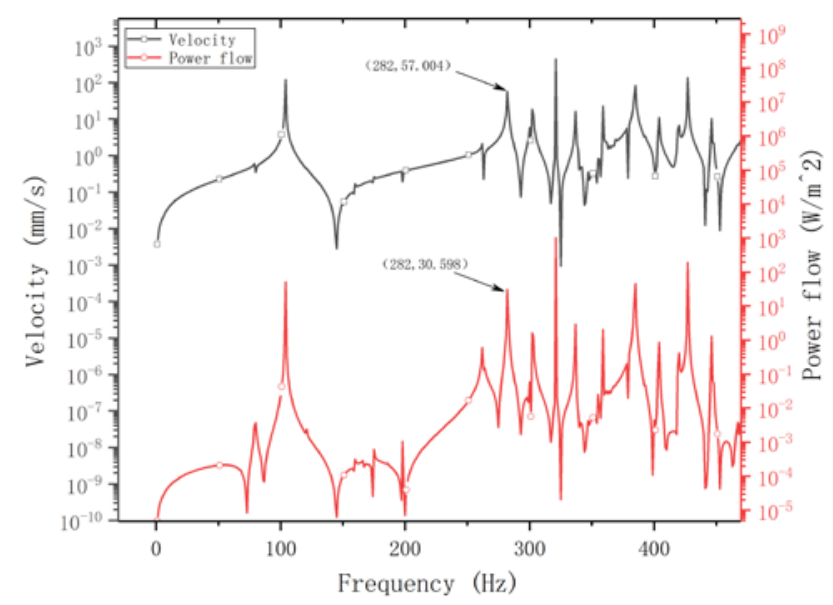

Fig. 5. Vibration velocity and power flow of the backup roll

From Fig. 3 to Fig. 5, we can see that the vibration speed harmonic response spectrum of upper working roll, upper intermediate roll and upper backup roll are quite similar with that of vibration power flow harmonic response spectrum, but there is no dominant frequency with $282 \mathrm{~Hz}$ in the vibration speed harmonic response spectrum of upper working roll, in the power flow harmonic response spectrum we can see a dominant frequency with $282 \mathrm{~Hz}$. Therefore, the traditional vibration velocity (displacement or acceleration) can't fully reflect the reality of the vibration energy.

\section{Visualization of power flow transfer}

\section{1 power flow vector visualization}

The visualization of power flow transfer path can clearly express the energy distribution, amplitude and flow direction in the research object. Zhu Xiang, Li Tianyun [14], et al used the finite element method to visualize the power flow of the cracked structure; in literature 15, the power flow finite element method was used to visualize and study the energy flow of the plate, the warehouse section and the whole ship in detail[15]. The above research shows that power flow visualization is an effective method to study energy transfer.

\subsection{1 visualization of rolling mill power flow vector}

The post-processing of finite element analysis shows that all nodes of the model have displacement solutions in three directions of $\mathrm{X}$-axis $\mathrm{Y}$-axis and $\mathrm{Z}$-axis in the global coordinate, and the displacement vector of each node is obtained with the displacement solution, and then obtain the displacement field of the whole model. Similarly, the displacement and stress solutions of each node in three directions can be obtained in the ANSYS postprocessor, and the power flow field of the whole model can be obtained by outputting the displacement solution and stress solution and then using the MATLAB color vector program developed independently. According to the Eq.1, the data of rolling mill at $104 \mathrm{~Hz}$ frequency are obtained from the post-processing of ANSYS, and the power flow vector of work roll, middle roll and backup roll is obtained as shown in Fig.6.

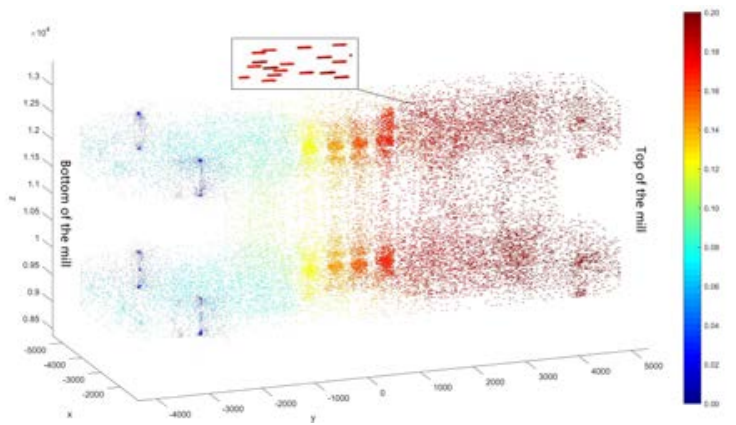

Fig. 6. Displacement vector diagram of rolling mill

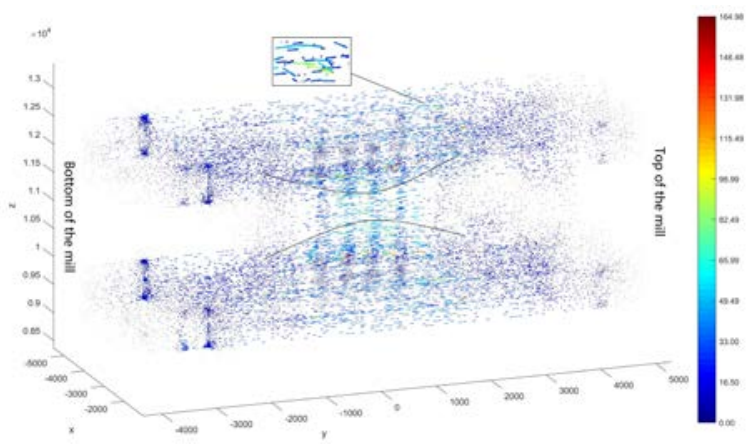

Fig. 7. Power flow vector diagram of rolling mill 
From Fig.6, we can see that the displacement vector of the rolling mill at the frequency of $104 \mathrm{~Hz}$ increases gradually from the bottom of the rolling mill (left side in Fig.6) to the top (right side in Fig.6). The displacement vector above the upper backup roll of the rolling mill is similar in size. The partial enlarged figure in Fig.6 shows that the whole displacement vector flows from the bottom of the rolling mill to the top.

According to Fig. 7, the power flow vector and displacement vector of the rolling mill at the frequency of $104 \mathrm{~Hz}$ are totally variable. The power flow distributed in the working roll and intermediate roll of the rolling mill is larger than that in other parts; the flow direction of power flow is not a single flow from the bottom of the rolling mill to the top, and the power flow at the roll system presents a similar hyperbolic flow path (as shown in the identification line in Fig.7), while the power flow of the house column directly flows from the bottom of the rolling mill to the top.

\subsection{2 visualization of roll system power flow vector}

Displacement vector diagram and power flow vector diagram of the upper roll system of the rolling mill with the frequency of 104Hz are shown in Fig.8 and Fig.9.

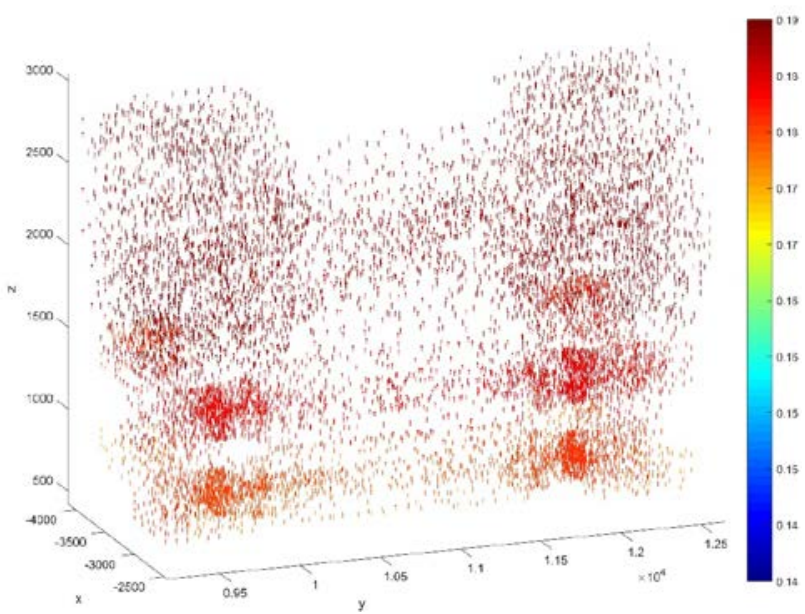

Fig. 8. Displacement vector diagram of upper roll system with the frequency of $104 \mathrm{~Hz}$

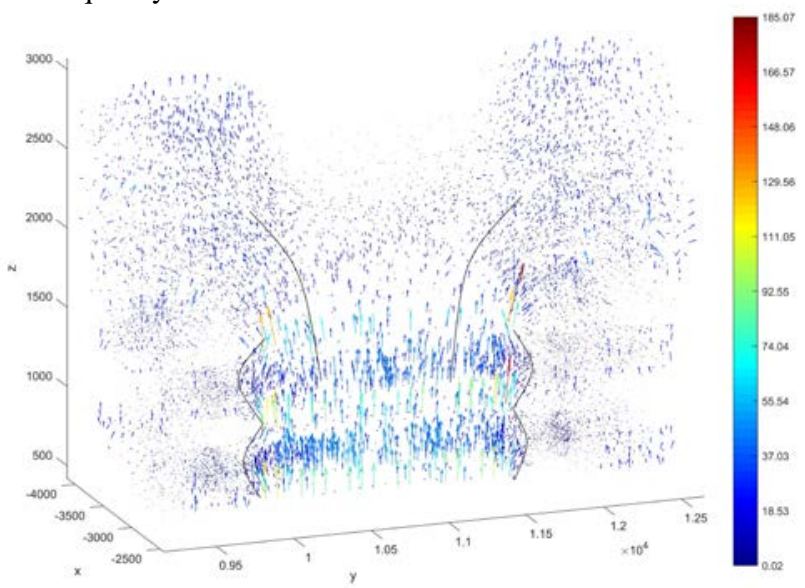

Fig. 9. Powerflow vector diagram of upper roll system with the frequency of $104 \mathrm{~Hz}$

From Fig.8 the vibration displacement direction of the upper roll system is basically identical, all of which are in the y-axis direction. The displacement vector amplitude of the backup roll is the largest, followed by the intermediate roll, and the working roll is the smallest. From Fig.9 we can see a great difference between the power flow vector and the displacement vector of the rolling mill model. The power flow vector can directly reflect the magnitude and flow direction of the vibration energy. The amplitude of power flow in the middle of work roll is much larger than that in the back-up roll. The power flow at the contact position of each roller is obviously larger than that at other postions, and the power flow at both ends of the contact surface between the rolls is larger than that in the middle part; the power flow amplitude at the outer end of each roller bearing seat is larger than that in the inner part of the bearing seat. When the power flow of the work roll and the intermediate roll is vertically upward from the bottom, the power flow will gradually diverge to the two ends with the size decreasing gradually after entering the upper backup roll; the power flow of the two sides of the intermediate roll and the upper work roll flows in a wave shape (as shown in the identification line in Fig.9).

The vibration displacement vector diagram and power flow vector diagram of the upper roll system of the rolling mill with the frequency of $283 \mathrm{~Hz}$ are shown in Fig. 10 and Fig.11.

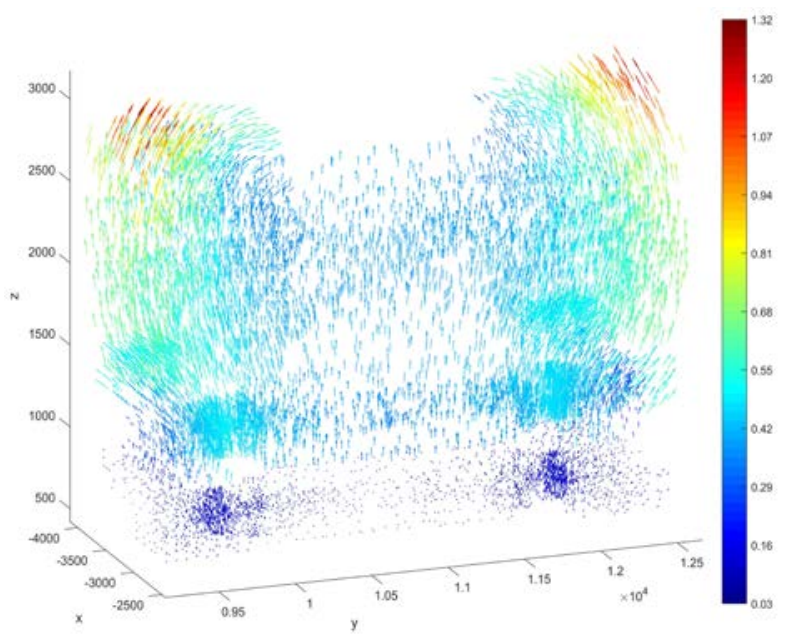

Fig. 10. Displacement vector diagram of upper roll system with the frequency of $283 \mathrm{~Hz}$

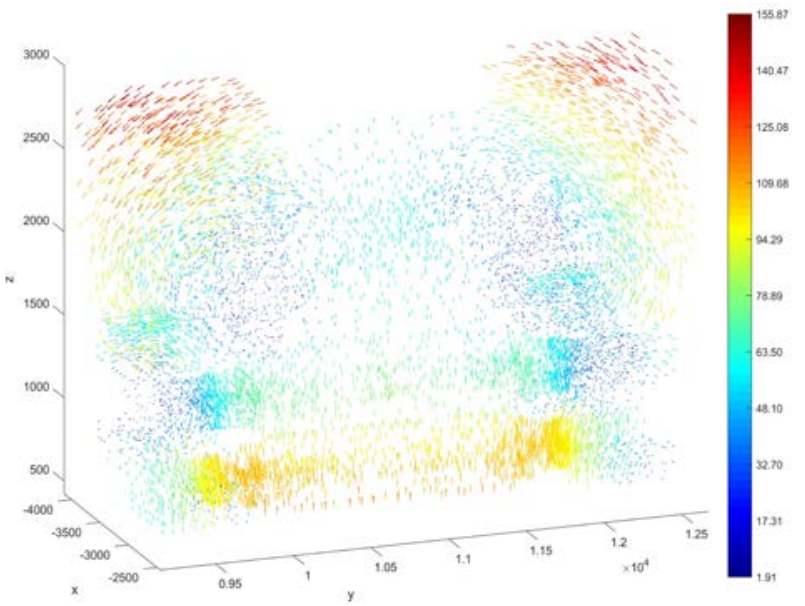

Fig. 11. Powerflow vector diagram of upper roll system with the frequency of $283 \mathrm{~Hz}$ 
From Fig.10, we can see that the displacement of the working roll with the frequency of $283 \mathrm{~Hz}$ is very small, and the displacement of the intermediate roll and the backup roll is large. However, the vibration power of the working roll is obviously larger than that of the intermediate roll and the backup roll (excluding the bearing seat of the supporting roll) as shown in Fig.11, and the vibration power is transmitted vertically upward. Compared with Fig.10 and Fig.11, we can see that the displacement vector of the bearing seat of the supporting roller is transferred in an arc from the bottom to the top, while the power flow vector is transferred in an arc from the top to the bottom, the transfer direction of the two is opposite.

\section{Conclusion}

1) Finite element harmonic response of vibration velocity can't reflect the real situation of vibration energy: the information of a large vibration energy fluctuation with a tiny vibration displacement is not clearly in the traditional vibration velocity harmonic response spectrum. However, the power flow harmonic response spectrum can fully reflect the vibration energy situation of the rolling mill.

2) The displacement vector of the traditional finite element harmonic response can't accurately reflect the actual vibration energy and flow direction of the rolling mill: the displacement vector amplitude of the rolling mill gradually increases from the bottom of the rolling mill to the top of the rolling mill with the frequency of $103 \mathrm{~Hz}$ and presents a single direction flow path. With the power flow vector visualization, we found that the vibration energy in the rolling mill does not flow in a single direction, the power flow in the rolls presents a flow path of approximate hyperbola, and the power flow at the end of the rolls presents a wave shaped flow, while the power flow in the house column of the rolling mill is transmitted vertically from the bottom to the top.

\section{References}

1. R. Lyon, G. Maidanik. J. Acoust. Soc. Am. 34, 623(1962)

2. H. Goyder, R. White. J. Sound Vibr. 68 59(1980)

3. H. Goyder, R. White. J. Sound Vibr. 68 77(1980)

4. H. Goyder, R. White. J. Sound Vibr. 68 97(1980)

5. R. Pinnington, R. White. J. Sound Vibr.75 179(1981)

6. K. Song, W. Zhang, J. Niu. CHIN J MECH ENG 09 23(2003)

7. B. Xiao, B. Li, C. Xia. CHIN J MECH ENG, 47 106(2011)

8. W. Zhang, J. Niu, J. Song. CHIN J MECH ENG 01 129(2007)

9. X. Wang, L. Sun, Y. Gao. CHIN J MECH ENG 51 48(2015)

10. Y. Cai, F. Zeng, J. Liu. J VIB SHOCK, 37 60(2018)

11. N. Fu, Y. Liu, Z. Zhao. CHINA J RAIL SOCI, 40 111(2018)

12. J. Liu, G. Lai, Y. Cai. CHINA J SHIPBUILD,58 68(2017)

13. L. Gavri, G. Pavi. J. Sound Vibr, 164 29(1993)

14. X. Zhu, T. Li, Y. Zhao, J. Liu. CHIN J MECH ENG, 45:132(2009)

15. Z. Wu, B. Xie. CHINA J RAIL SOCI, 2295 (2016) 